\title{
Determination of the patch cords lengths distribution physical layer of the modern automation systems of smart city and smart home by the Monte Carlo method
}

\author{
Andrey Semenov \\ Moscow State University of Civil Engineering, Yaroslavskoe shosse, 26, Moscow, 129337, Russia
}

\begin{abstract}
A method for determining the distribution of patch cord lengths for the formation of the physical layer of modern IP-control systems is proposed. Assuming the formation of the switching field on a regular basis and the equiprobable connection of individual ports, a procedure was developed for determining the probability density function of the cord lengths, taking into account the necessary margin in case of unevenness of the laying. The calculation of the distribution has been performed and it is shown that it is advisable to use patch cords with a length of $1-2 \mathrm{~m}$ in control systems.
\end{abstract}

\section{Introduction}

One of the main requirements of permanent residents and guests of modern urbanized areas is to ensure a certain level of comfort, which is achieved by the introduction of a large number of engineering systems both at the city level and in individual residential and public buildings $[1-4]$. The greatest effect of the operation of such systems is achieved if they are operated as a single complex, which, in turn, requires the creation of an automated control system for both the entire city and individual buildings. At a certain level of integration of the control system with the serviced object, a qualitative transition occurs and the latter turns into an intelligent one [5-7].

The Smart Home automation system as an integral part of Smart City [8, 9], regardless of scale, is built in accordance with the time-tested model of open systems. The physical layer as a basic component can be implemented in different ways, but in most cases this involves wired communication channels, which, in accordance with the requirements of the standards, are implemented in the form of SCS (Structured Cable System).

\section{Research objective}

SCS as a system can contain two main parts: line cables and switching equipment, with the help of which various active network devices are connected to the cables [10]. Individual line cables are led to technical rooms, where they are connected to group patch panels. When forming transmission channel, the ports of the panels and LAN switches are connected to 
each other. The combination of panels, switches and auxiliary elements forms a switching field.

Port connection can be done by switches or patch cords, Fig. 1.
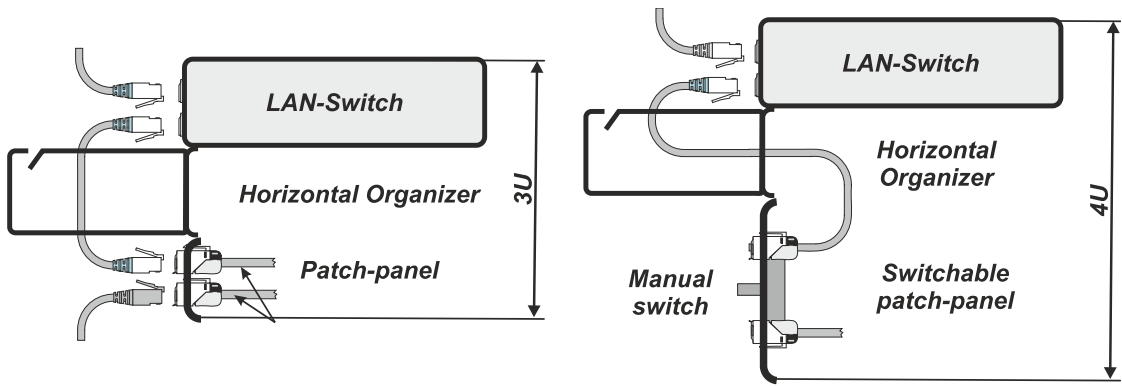

Fig. 1. Panel and switch port connection options: traditional cords (left) and built-in panel manual or automatic switches (right)

Switch panels are approved for use by ISO/IEC 11801-1:2019 [11]. The switch state can be changed manually or automatically. Such panels are very rare. They are only seriesproduced by the Israeli company RiT Technologies. The reason for this:

- low functional flexibility, because the switch works only for two ports located one above the other;

- panels allow to create a switching field only according to the cross-connect scheme, which reduces its resultant density.

Therefore, in the future, we will focus only on the second option: connecting ports with patch cords.

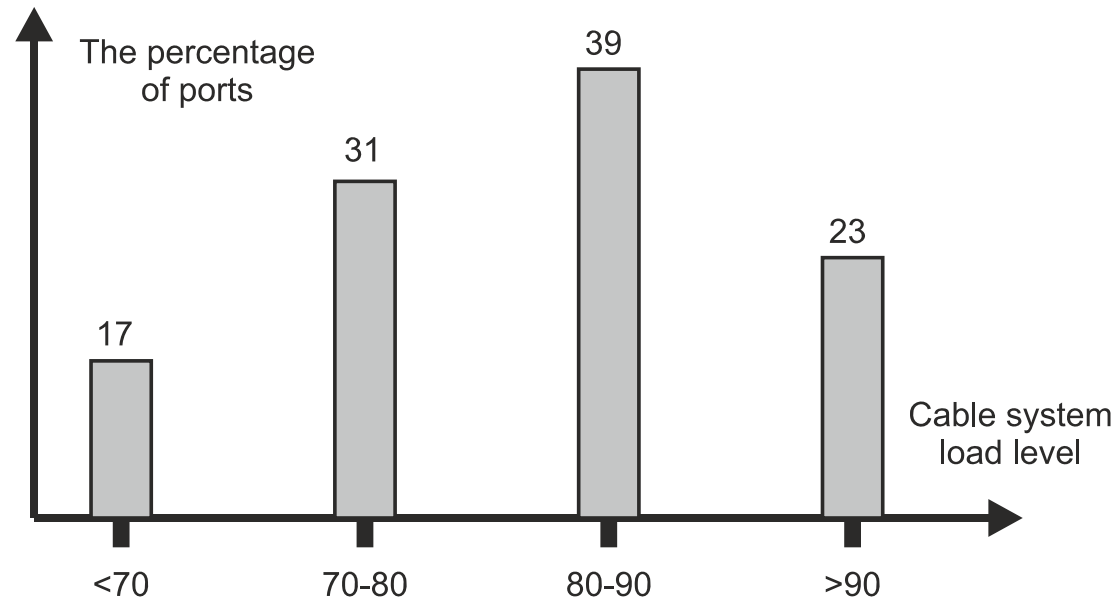

Fig. 2. The SCS resources load rate of large automation systems for smart homes

Manufacturers produce copper patch cords of various lengths and limited nomenclature. At the same time, European companies gravitate to the decimeter sample of this parameter, i.e. $0.5 \mathrm{~m}, 1.0 \mathrm{~m}$, etc., and American - to the foot sample, i.e. $0.6 \mathrm{~m}, 0.9 \mathrm{~m}, 1.2 \mathrm{~m}$, etc. The shortest cords should be used, when forming the transmission channel. This improves two key parameters for the cable system operation.

- the quality of information transmission over the cable channel (attenuation of the patch cord cable with stranded wires is $1.2-1.5$ times higher than that of the rigid installation cable with solid wires) $[12,13]$; 
- the cable system administration conditions due to the absence of loops $[14,15]$.

An effective solution to these problems is extremely complicated by a high level of resource utilization, which is typical for modern structured cable systems Fig. 2. This requires the use of patch cords of various lengths, the number of which is subject to a certain distribution. The purpose of this work is to determine such distribution.

\section{Rules for defining the switching field and mathematical model}

Further analysis assumes

- the information system is implemented entirely on the basis of IP technology [16];

- the switching field is built on separate functional sections, each containing only the same-type equipment [17];

- the functional section equipment is housed in an $800 \mathrm{~mm}$ wide 19-inch cabinet; [18 19];

- patch panels and switches have a $1 \mathrm{U}$ of height case and a 24 port construction density per $1 \mathrm{U}$ height;

- existing differences in the form factor of panels and switches are considered negligible;

- for switching, patch cords of the minimum possible length are used, switching between different ports is equally probable [20];

- switching is carried out only between panels and switches, i.e. between different functional sections of the switching field;

- functional sections on both sides are supplemented with vertical cable organizers;

- every two panels/switches of the functional section are complemented by a horizontal organizer [21].

The latter increases the efficiency of administration due to the fact that after connecting the patch cord cable to the panel/switch port, it immediately goes into the organizer and does not cross the adjacent row of outlets.

The switching field implementing these principles is shown in Fig. 3.

From the right side of Fig. 3 it follows that regardless of the connected ports for any patch cord, can be written as

$$
L=1.1 \cdot(\mathrm{Hor}+\mathrm{Ver})
$$

where Hor - a horizontal part of the cable;

$\mathrm{Ver}$ - a vertical part of the cord;

1.1 - an empirical margin for unevenness of laying, connecting a patch cord to patch panels and likewise.

Taking into account the random nature of the switching field individual ports connection for the Fig. 3 structure using the notation system introduced there for (1) we have

$$
\begin{aligned}
& \text { Hor }=\min [\xi+\mu ; 642-(\xi+\mu)], \\
& \operatorname{Ver}=\zeta+\eta+\delta_{\zeta}+\delta_{\eta},
\end{aligned}
$$

where: $\delta_{\zeta}, \delta_{\eta}=0$ or $\delta_{\zeta}, \delta_{\eta}=44,45 \mathrm{~mm}$ depending on the actual value $\eta$ and $\zeta$. 

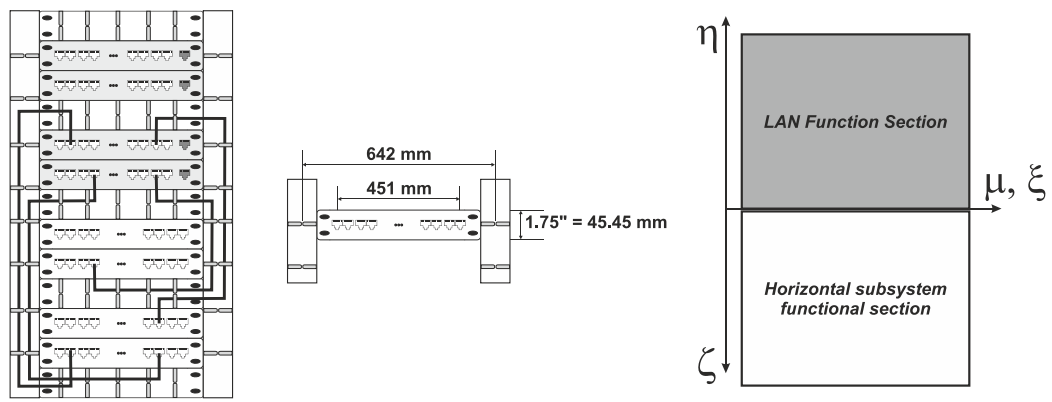

Fig. 3. The switching field definition and the patch cords routes (on the left), the dimensions used in the calculations (in the center), the coordinate system (on the right)

The operator's action scheme (2) is illustrated in Fig. 4.

Note that the absolute values $\zeta, \eta$ are counted from the origin of coordinates in opposite directions. Placing the origin of coordinates between the functional sections simplifies calculations.
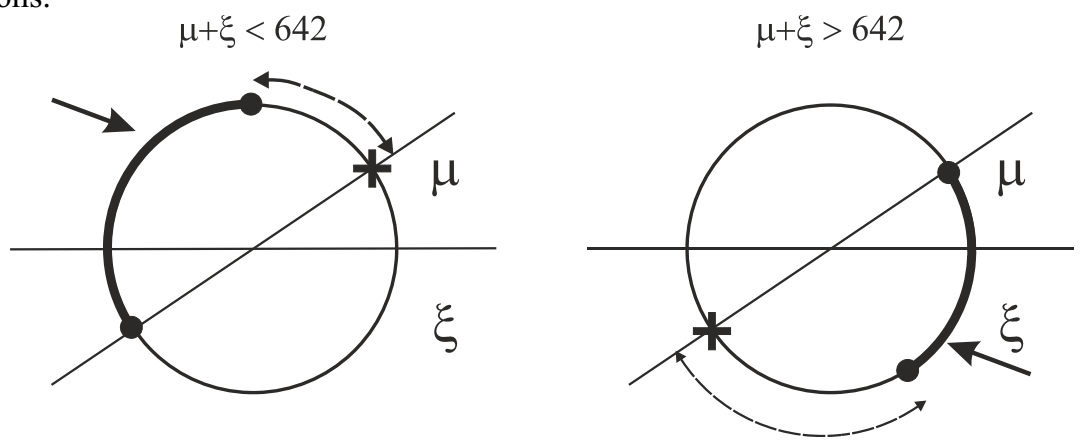

Fig. 4. Scheme for choosing one of two values $\xi+\mu$ and $642-(\xi+\mu)$

\section{Analysis of the mathematical model}

From Fig. 4 it follows that the random variables $\xi+\mu$ and $642-(\xi+\mu)$ with an equal probability of their occurrence are distributed in the same way. The latter means that, taking into account (1), the desired distribution is a composition of the following form

$$
\xi+\mu+\zeta+\eta+\delta_{\zeta}+\delta_{\eta}
$$

$\xi, \mu, \zeta, \eta$, according to the introduced model, have a uniform distribution, and $\delta_{\zeta}, \delta_{\eta}-$ are discrete random variables that take two values: 0 or $1 \mathrm{U}$.

The sum (3) is a composition of independent random variables, the probability density function of which is calculated as their convolution. In particular, this function is easily calculated analytically for the $\operatorname{sum} \xi+\mu$ considering the limitation $\xi+\mu<642 \mathrm{~mm}$

$$
\varphi_{\xi+\mu}(x)=\int_{-\infty}^{\infty} \varphi_{\xi}(\tau) \cdot \varphi_{\mu}(x-\tau) d \tau,
$$


is presented in Fig. 5 and considering the specified 19-inch cabinet format does not depend on the number of serviced terminal devices.

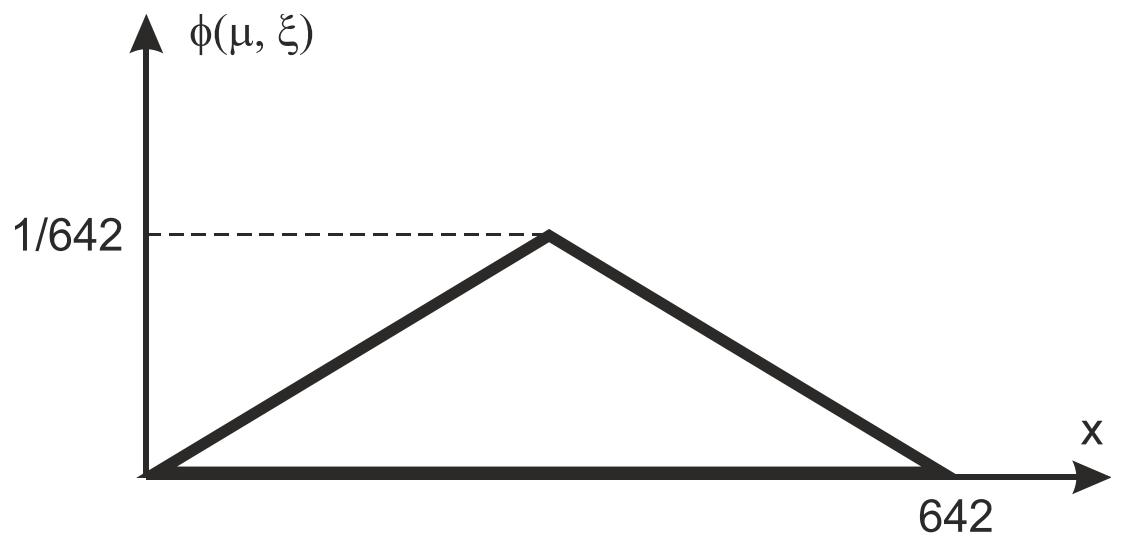

Fig. 5. The probability density function of a random variable $\xi+\mu$

Analytical determination of the probability density function of the entire composition $\xi+\mu+\zeta+\eta+\delta_{\zeta}+\delta_{\eta}$ is complicated by the presence of $\delta_{\zeta}+\delta_{\eta}$ the sum of two independent discrete components in its composition. The result of the calculation is a cumbersome expression, poorly suited for use in general engineering practice. In $[7,8]$, this complexity was eliminated by approximating the resulting distribution of the Gaussian curve, the variance of which coincides with the variance of the sum $\xi+\mu+\zeta+\eta$ with the additional assumption of the variance of the sum smallness $\delta_{\zeta}+\delta_{\eta}$ and the equality of its mathematical expectation $2 U=88.9 \mathrm{~mm}$.

From Fig. 4 it follows that the composition distribution $\xi+\mu$ is very different from normal, which leads to a large approximation error. To eliminate this drawback, let us turn to mathematical modeling using the Monte Carlo method.

\section{Features of the task $\eta+\delta_{\eta}$ and $\zeta+\delta_{\zeta}$}

For the calculation, the classical implementation of the Monte Carlo method was used, and the built-in Excel spreadsheet operator with the appropriate scaling of the form $y=k \cdot x$, was used as a random number generator where $x$ is evenly distributed over the interval $[0 ; 1]$, and $k$ is numerically equal to the maximum value of the corresponding random variable. So, for $\xi$ and $\mu$ based on Fig. 3 we have $k=642, k=\max n$ can be taken for $\eta$ and $\zeta$ where $n$ depends on the number of serviced terminal devices and is further determined.

Direct application of the operator $y=k \cdot x$, to the task $\eta$ and $\zeta$ is incorrect due to the presence of horizontal organizers on switching field. To eliminate the error created by its presence, the following procedure was applied:

Using the operator $y=k \cdot x$, with additional operator processing CEILING the preliminary conditional number $\mathrm{n}$ of the panel/switch is found

$$
n 1=C E I L I N G(k \cdot x)
$$


The actual number of the panel, taking into account the organizers and the regular nature of the switching field definition, will be

$$
\begin{aligned}
& n=1 \text { at } n 1=1 \\
& n=\operatorname{CEILING}(n 1 / 2-1)+n 1 \text { at } n 1>1
\end{aligned}
$$

The values $\eta$ and $\zeta$ in the coordinate system Fig. 3 are determined as

$$
\begin{gathered}
\eta=44.45 \cdot n_{\eta}, \\
\zeta=\left(N-n_{\zeta}\right) \cdot 44.45,
\end{gathered}
$$

$N$ - total height of the horizontal subsystem functional section.

The principle of determining the actual panel number in graphic form is illustrated in Fig. 6.

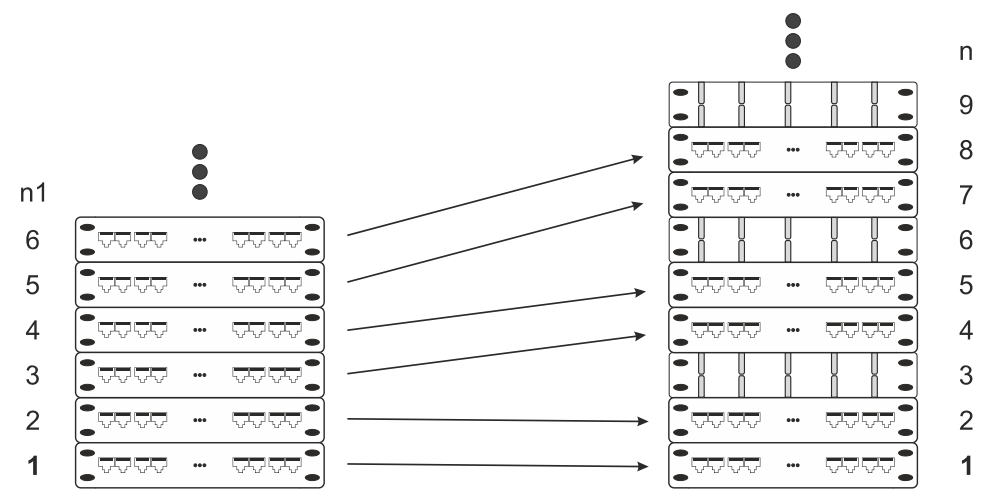

Fig. 6. The principle of renumbering panels taking into account horizontal organizers

When defining parameters $\delta_{\eta}$ and $\delta_{\zeta}$ we proceed from the fact that $\delta_{\eta}=44.45 \mathrm{~mm}$ with odd $n_{\eta}$ and $\delta_{\eta}=0$ with even $n_{\eta}$, whereas $\delta_{\zeta}=44.45 \mathrm{~mm}$ with even $n_{\zeta}$ and $\delta_{\zeta}=0$ with odd $n_{\zeta}$. Formally, for example, for $\delta_{\zeta}$ it can be written as

$$
\operatorname{IF}\left[\left(\operatorname{CEILING}\left(n_{\zeta} / 2-n_{\zeta} / 2\right)=0\right) \operatorname{THEN} \delta_{\zeta}=0 ; \delta_{\zeta}=44.45\right] .
$$

\section{Determination of the proportion of cords of a certain length and calculation results}

The above procedures with the additional involvement of (1) make it possible to determine the desired distribution $\varphi(x)$.

If the SCS manufacturer offers an ordered range of cords with certain lengths $a, b, c \ldots$ wherein $a<b<c<\ldots$ Then the share of a particular type of cords is

$$
\int_{0}^{b} \varphi(x) d x-\int_{0}^{a} \varphi(x) d x .
$$


The calculation results using (4) are shown in Fig. 7. In the process of numerical modeling using a random number generator, a set of 20 values of random vectors $(\xi ; \mu ; \zeta ; \eta)$ was formed followed by averaging. The maximum number of $N$ terminal devices serviced by SCS also varied.
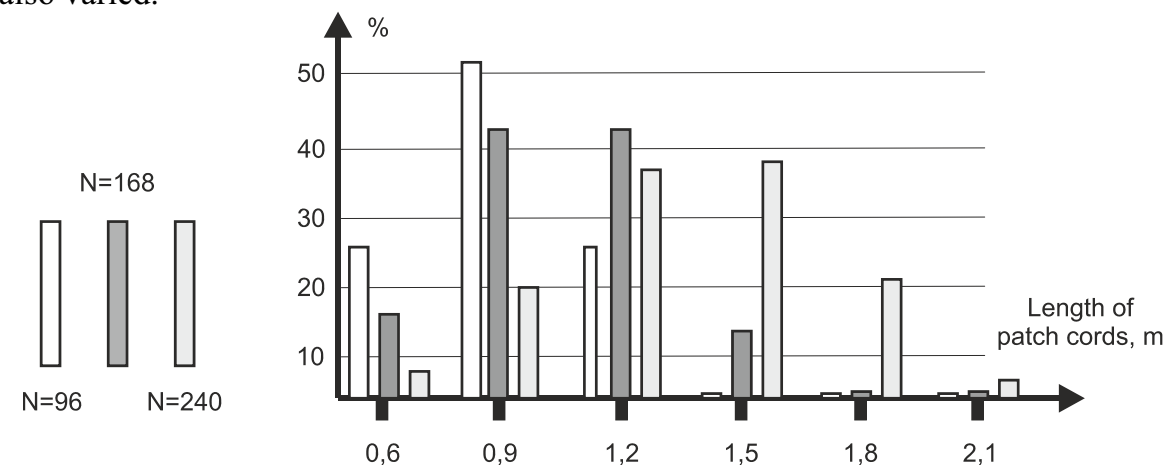

Fig. 7. The share of cords of various lengths used during the SCS operation with a different number of ports

The diagram on Fig. 6 is of a general nature and allows solving the problem of choosing the distribution of cords for a typical case of constructing an automatic control system. This is due to the fact that, according to available statistics, the average number of serviced terminal devices in a modern control system amounts approximately to 140 .

\section{Conclusion}

The most popular cords in the technical rooms of control systems are not 1-2 m long.

Short $60 \mathrm{~cm}$ cords are in demand only in small control systems with the number of serviced terminal devices not exceeding 100-120.

The problem was solved using general-purpose software, and performing calculations does not require large computational tasks.

Taking into account the small number of input parameters, the proposed method can easily be extended to free-hand switching field configurations on the 19-inch equipment, provided that its structure is formed regularly.

\section{References}

1. A. Volkov, Industrial and civil engineering 94 (2017)

2. A. Volkov, Industrial and civil engineering 96 (2019)

3. A. Volkov, Industrial and civil engineering 1190 (2013)

4. A. Volkov, P. Chelyshkov, A. Sedov, Industrial and civil engineering 11264 (2012)

5. N. Stefanova, Current issues of the modern economy 4175 (2018)

6. N. Stefanova, Issues of sustainable development of society 624 (2018)

7. S. Shylkina, A. Gusarova, Scientific review 2247 (2016)

8. A. Semenov, LAN Mag Russian Edition 1028 (2017)

9. P. Chelyshkov, A. Semenov, Vestnik svjazi 24 (2019)

10. A. Semenov, Last mile 332 (2019) 
11. ISO/IEC 11801 - 1:2019. Information technology - Generic cabling for customer premises - Part 1: General requirements, International standard. 2019164

12. M. Bylina, S. Glagolev, A. Semenov, R. Davydov, Proceedings of the 2020 IEEE International Conference on Electrical Engineering and Photonics, EExPolytech 2020, 9243954 ,

13. A. Semenov, J. of Physics: Conference Series, 1582(1), 012075 (2020)

14. A. Semenov, IOP Conference Series: Materials Science and Engineering, 869(2), 022022, (2020)

15. A. Semenov, Proceedings - 2020 International Conference on Industrial Engineering, Applications and Manufacturing, ICIEAM 2020, 9112002 (2020)

16. J. Rech, Ethernet, Technologien and Protokolle fuer die Computervernetzung, Heise 550 (2008)

17. A. Semenov, Design and calculation of structured cabling systems, Profobrazovanie, Saratov 416 (2017)

18. B. Elliot Design a structured cabling system to ISO $118012^{\text {nd }}$ edition, Cambridge 316 (2010)

19. I. Smirnov, Structured cabling system - design, installation and certification, EkonInform, Moscow 348 (2005)

20. P. Vorobienko, A. Nikitiuk, P. Reznichenko Telecommunication and information networks, Sammit-kniga, Kiev 632 (2010)

21. J. Dittrich, U. von Thienen, Moderne Datenverkabelung, ITP, Bonn 518 (1998) 